\title{
Ion beam induced charge (IBIC) irradiation damage study in synthetic single crystal diamond using $2.6 \mathrm{MeV}$ protons
}

\author{
A. Lohstroh ${ }^{* 1}$, P. J. Sellin ${ }^{1}$, S. Gkoumas ${ }^{1}$, J. Parkin $^{1}$, P. Veeramani ${ }^{1}$, G. Prekas ${ }^{1}$, M. C. \\ Veale $^{1}$, and J. Morse ${ }^{2}$ \\ ${ }^{1}$ Department of Physics, University of Surrey, Guildford, GU2 7XH, United Kingdom \\ ${ }^{2}$ European Synchrotron Radiation Facility (ESRF), 6 rue de Jules Horowitz, BP 220, 38043 Grenoble, \\ Cedex, France
}

Received zzz, revised zzz, accepted $z z z$

Published online $z Z z$

PACS 29.40.Wk, 72.20.Jv, 61.80.-x

\begin{abstract}
We have studied the effects of irradiation induced damage on the detector response of a synthetic single crystal diamond radiation detector. Before introducing radiation damage, the spatial variation of the detector response was investigated using a highly focused $2.6 \mathrm{MeV}$ proton beam. A very uniform response close to $100 \%$ charge collection efficiency (CCE) over the whole contact area was found at applied elec-

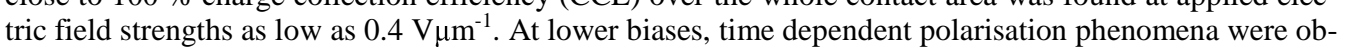
served and clearly reduced the average signal amplitude. Subsequently, the $2.6 \mathrm{MeV}$ proton beam has been used to introduce radiation damage within selected areas. The ion beam induced charge imaging was

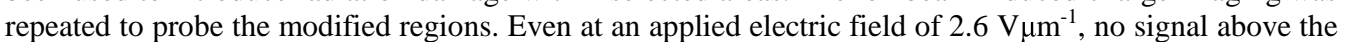
analogue threshold of the system was observed in the areas which had received a dose larger then $5 \times 10^{14}$ $\mathrm{cm}^{-2}$, whereas more than $90 \%$ CCE was reached in the area with $10^{12}$ protons $\mathrm{cm}^{-2}$.
\end{abstract}

\section{Introduction}

Diamond has been of interest for room temperature radiation detection applications for many decades, due to the low intrinsic conductivity of high purity material, which provides low leakage current induced noise levels. Recent studies have shown excellent spectroscopic and timing performance of devices based on synthetic high quality chemical vapour deposited (CVD) single crystal diamonds produced by Element Six Ltd., UK [1]. This material is superior to polycrystalline diamond and most natural stones due to its high purity and low dislocation concentration, exhibiting high mobilities [2]. It has intrinsically long charge carrier lifetimes that determine detector performance and does not need to be pre-irradiated (primed) before operation to increase charge carrier lifetime like most polycrystalline devices [3].

Diamond is also known for its high thermal conductivity, chemical inertness and hardness - including radiation hardness, which makes it very attractive for applications in high radiation fluence environments [4]. The defects caused by light ion irradiation or implantation are mainly thought to be vacancies and interstitials [5], which are not mobile at room temperature [6]. Radiation damage effects in polycrystalline diamond detectors only result in a noticeable reduction of charge collection distance at an applied

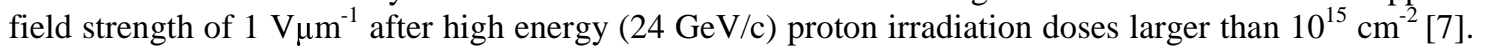
Investigations of the degradation of charge transport in high quality CVD single crystals have begun more recently. It has been found that the detector signals reduce noticeably after $26 \mathrm{MeV}$ proton irradiation of $5 \times 10^{14}$ protons $\mathrm{cm}^{-2}$ [8]. Absorption studies have shown an increase in the neutral vacancy and vacancy-interstitial related cluster signals; the charge carrier lifetime in the non-primed state was reduced by more than an order of magnitude, but could be increased by priming, whereas the charge carrier velocity was not affected by the introduced damage [9]. Unfortunately, it is still not well understood, if and

* Corresponding author: e-mail: A.Lohstroh@surrey.ac.uk, Phone: +44 148368 9422, Fax: +44 1483686781 
which of the introduced defects most crucially act as charge trapping centre reducing the charge carrier lifetime as well as causing polarisation and priming.

\section{Experimental details}

The single crystal synthetic diamond plate with dimensions of $3.5 \times 3.5 \times 0.38 \mathrm{~mm}^{3}$ was purchased in 2006 from Element Six Ltd., who synthesise the material with a microwave plasma enhanced CVD process [2] and have subsequently resin wheel and scaife polished it. It has been cleaned in aqua regia followed by a wash in acetone and isopropanol before it was contacted with a simple sandwich pad structure of $50 \mathrm{~nm}$ gold layers on both sides, deposited by thermal evaporation.

The ion beam induced charge (IBIC) imaging was performed at the Surrey Microbeam line [10], using 2.6 MeV protons with a typical beam current of less then $1 \mathrm{fA}$ and a beam spot size of less than $10 \mu \mathrm{m} \mathrm{x}$ $10 \mu \mathrm{m}$. The protons penetrate approximately $37 \mu \mathrm{m}$ into the diamond as calculated by SRIM (http://www.srim.org)[11] through the biased top contact. Therefore, the induced integrated current signal at positive bias is mainly due to hole transport and the signal collected at negative bias originates mainly from electron movement. The current signal of the detector is integrated by a charge sensitive pre-amplifier (Ortec $142 \mathrm{~A}$ ). The signal is passed on to a shaping amplifier (Ortec 570) and subsequently digitised and saved pulse by pulse with its respective beam position. Charge collection efficiency (CCE) images are calculated as the mean induced amplitude of all registered events per pixel. The energy to create an electron hole pair in diamond $\mathrm{W}_{\text {ehp }}$ was assumed to be $13.2 \mathrm{eV} \mathrm{[12],} \mathrm{which} \mathrm{is} \mathrm{in} \mathrm{agreement}$ within $3 \%$ of other publications $[1,13,14]$ and our own particle induced charge pulse studies. Some publications suggested $\mathrm{W}_{\text {ehp }}$ values deviating from $13 \mathrm{eV}$. A more detailed discussion can be found in the article by Kashiwagi et al. [15]. The acquired pulse height spectra (PHS) were calibrated with a pulser and cross correlated to a Silicon device, which was assumed to have $100 \% \mathrm{CCE}$ and $\mathrm{W}_{\text {ehp }}=3.6 \mathrm{eV}$ [16].

Table 1 Summary of the doses received by the selected areas of the device

\begin{tabular}{l||c|c|c|c|c|c}
\hline Label & $\mathrm{A}$ & $\mathrm{B}$ & $\mathrm{C}$ & $\mathrm{D}$ & $\mathrm{E}$ & $\mathrm{F}$ \\
\hline $\begin{array}{l}\text { Area } \\
{\left[10^{-3} \mathrm{~cm}^{2}\right]}\end{array}$ & $2.6 \pm 0.3$ & $2.4 \pm 0.3$ & $2.6 \pm 0.3$ & $0.6 \pm 0.14$ & $0.18 \pm 0.08$ & $0.05 \pm 0.04$ \\
\hline $\begin{array}{l}\text { Dose } \\
{\left[\mathrm{cm}^{-2}\right]}\end{array}$ & $\begin{array}{c}(1.1 \pm 0.1) \\
\mathrm{x} 10^{12}\end{array}$ & $\begin{array}{c}(1.1 \pm 0.1) \\
\mathrm{x} 10^{13}\end{array}$ & $\begin{array}{c}(1.0 \pm 0.1) \\
\mathrm{x} 10^{14}\end{array}$ & $\begin{array}{c}(5 \pm 1) \\
\mathrm{x} 10^{14}\end{array}$ & $\begin{array}{c}(1.0 \pm 0.4) \\
\mathrm{x} 10^{15}\end{array}$ & $\begin{array}{c}(5 \pm 4) \\
\mathrm{x} 10^{15}\end{array}$ \\
\hline
\end{tabular}

After the first set of IBIC images was acquired, selected rectangular areas labelled $\mathrm{A}$ to $\mathrm{F}$ have been irradiated with doses between $10^{12}$ and $5 \times 10^{15}$ protons $\mathrm{cm}^{-2}$. The same energy proton beam with an increased beam current, which required an increase in spot size to $45 \mu \mathrm{m} \times 90 \mu \mathrm{m}$, was used for this purpose. The device was not biased during these irradiations and the sample had to be exposed to light for sample positioning before the IBIC characterisation was repeated on the following day. The total dose received by each area is summarised in table 1 . The larger relative error in the high doses compared to the low ones reflects the larger uncertainty in the irradiated area size due to the beam dimensions.

\section{Results}

Fig. 1 (a) displays a CCE image with the colour scale ranging from $97 \%$ to $103 \%$ of the investigated sample at $+150 \mathrm{~V}$; essentially the same image was obtained subsequently at $-100 \mathrm{~V}$ bias supply and the corresponding PHS are shown in Fig.1 (b). The full width half maximum (FWHM) of the peaks centred around $100 \%$ CCE are $2.2 \%$ and $1.9 \%$ for electrons and holes respectively, which is about double of the values measured during the pulser calibration at the same amplifier settings. The absence of any significant lower energy tail in the spectrum demonstrates that at sufficiently high applied electric field, the detector essentially shows a $100 \%$ CCE response over the whole area, for electron and hole sensitive transport, with the exception of surface features. 

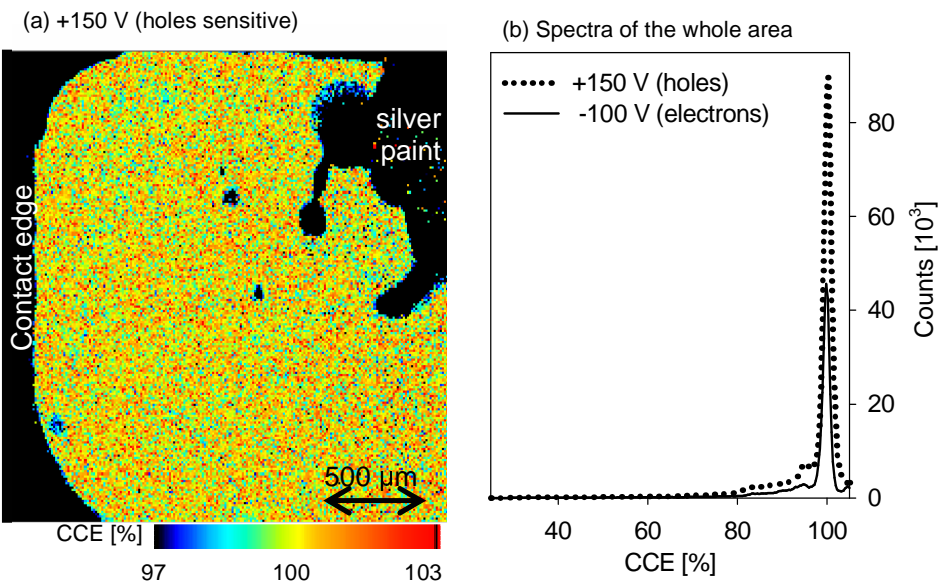

Fig. 1 (online colour at: www.pss-a.com) (a) CCE image of the device at high bias prior to the introduction of radiation damage, (b) PHS of the whole area displayed in (a) at high bias for hole and electron sensitive signals.

(a) $0 \mathrm{~s}$ to $162 \mathrm{~s}$ of the data file

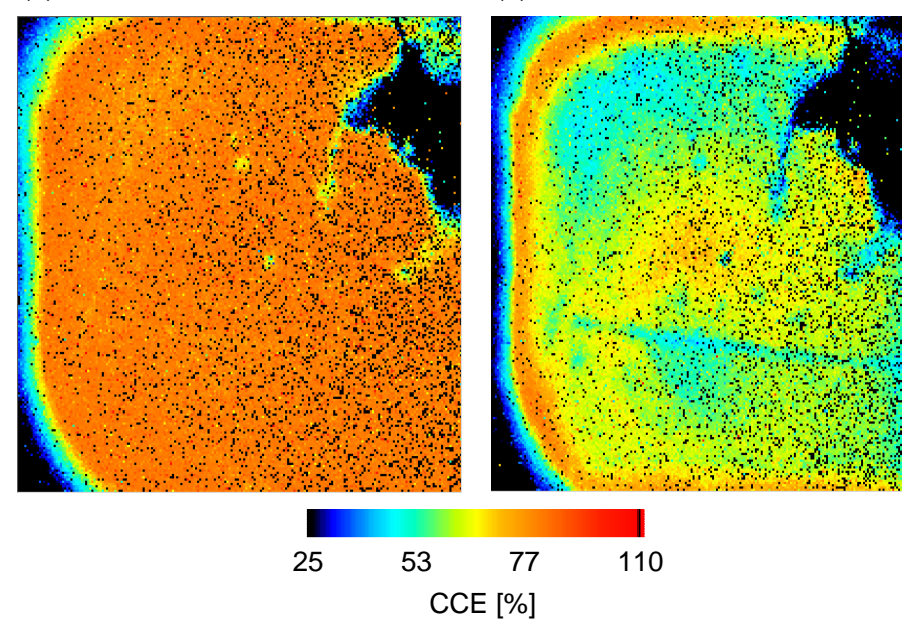

(c)

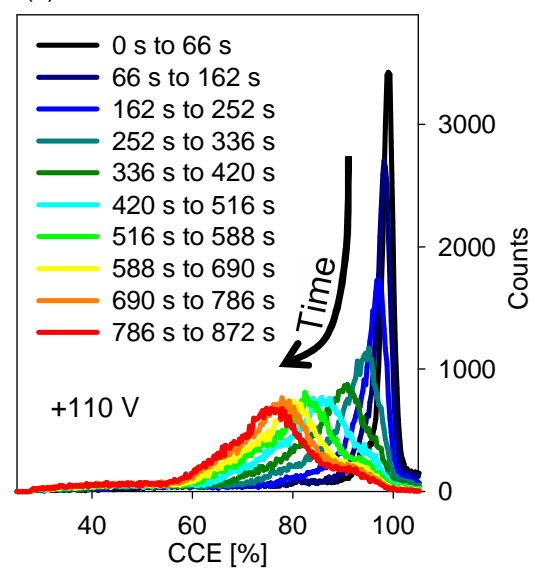

Fig. 2 (online colour at: www.pss-a.com) CCE image acquired at $+110 \mathrm{~V}$. (a) average CCE of the first $162 \mathrm{~s}$ of the measurement (b) average CCE of the last $182 \mathrm{~s}$ of the measurement (c) Time evolution of the PHS extracted of the scan area shown in (a) and (b).

Fig.2 (a) and (b) show the average CCE images extracted by analysing the first 162 s, i.e. one fifth of all acquired events, and the last $182 \mathrm{~s}$ of the dataset taken at $+110 \mathrm{~V}$. The random black pixels in the contacted area are most likely pixels which have not been hit by a proton during the analysed time interval. The deterioration of uniformity, decrease in average signal amplitude and broadening of the CCE spectrum is also illustrated in Fig. 2 (c) which displays the time evolution of the PHS during the measurement. At lower bias, the detector response does not only become non-uniform but also instable due to polarisation phenomena, which are well known in the literature for polycrystalline and single crystal devices [3, 17, 18]. However, in our case, these effects are overcome and become negligible at high applied electric field strength. It is not clear if this problem is caused by the material or by the contact quality. 
The areas which were deliberately irradiated with high beam currents to introduce damage, are all rectangular in shape and outlined by the dashed lines in Fig. 3 (d). Within the areas A, B, and C, smaller regions have been selected, which overlap with the irradiated part and the contact area. They are indicated in white and have been used to extract PHS before and after the damage was introduced. Fig. 3 (a) exemplifies one of these spectra extracted from the white rectangle within area B before damaging. The average CCE above the analogue threshold of the system has been calculated and is plotted as a function of voltage in Fig. 3 (b) and (c) for electrons and holes respectively. The data does not clearly follow the expected behaviour predicted by the Hecht equation [16], which is most likely due to the presence of internal electric fields caused by the polarisation effects discussed earlier.

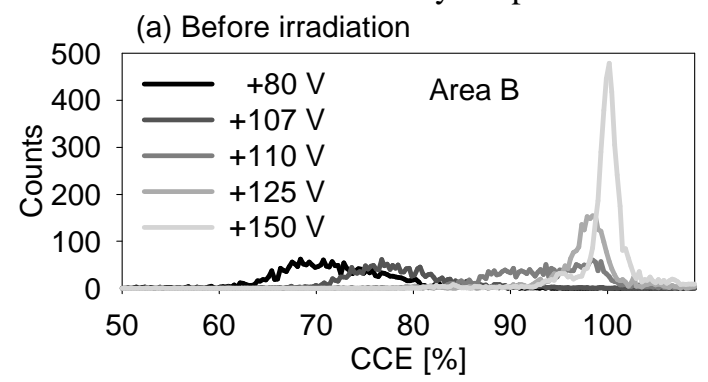

(d) After irradiation: $+150 \mathrm{~V}$
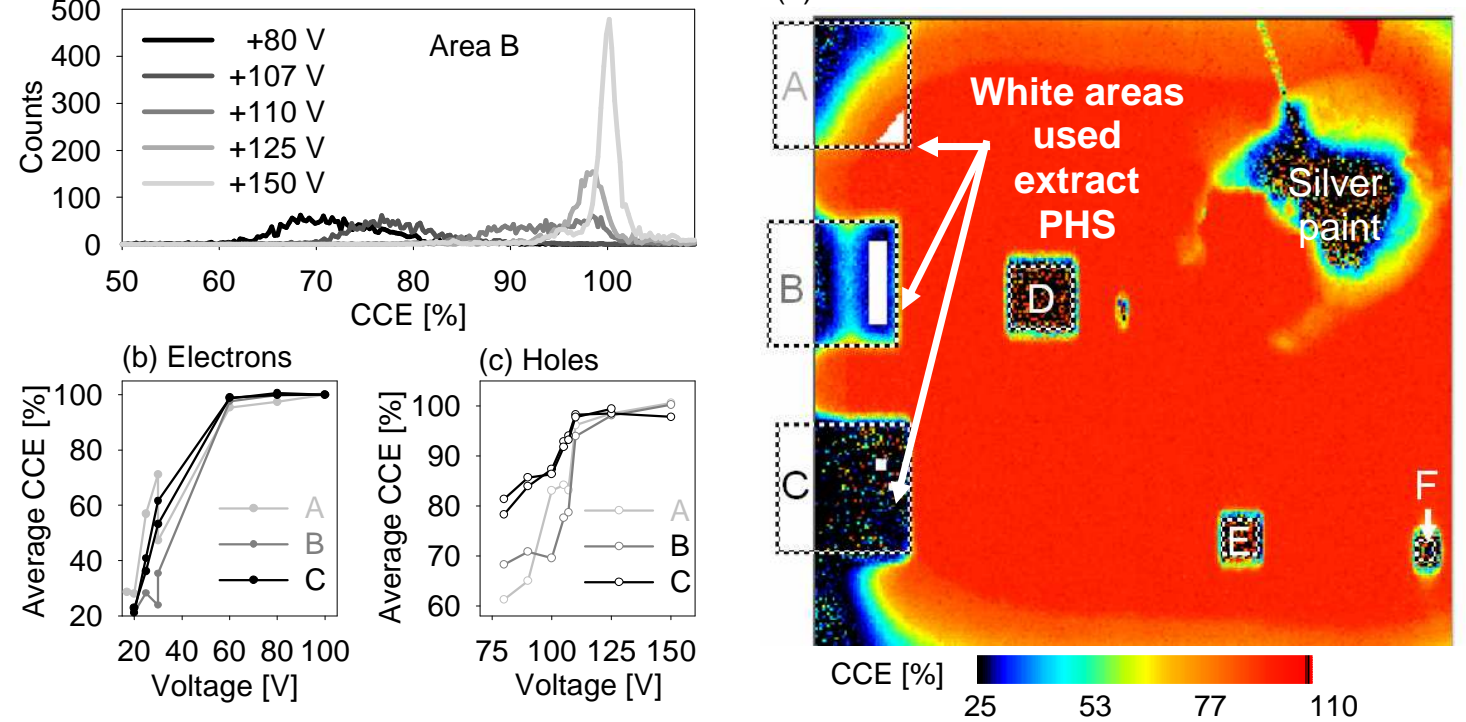

Fig. 3 (online colour at: www.pss-a.com) (a) PHS acquired from the white rectangular area within area B (indicated in Fig. 3 (d)), before irradiation damage was introduced. (b) and (c) Average CCE extracted from PHS similar to the one shown in (a) for electrons and holes respectively before irradiation. (d) CCE image acquired at $+150 \mathrm{~V}$ after the introduction of radiation damage in the areas $\mathrm{A}$ to $\mathrm{F}$.

Fig. 3 (d) displays the CCE image taken at $+150 \mathrm{~V}$ after radiation damage has been introduced into the areas A to F. The outlines of the irradiated rectangular areas can clearly be seen. A vertical strip of increased CCE is visible through area B, which we assign to a field enhancing effect at the edge of the metal contact; a similar effect can be observed in figure 2 (b). The areas D to F (irradiated with $\geq$ $5 \times 10^{14}$ protons $\mathrm{cm}^{-2}$ ) did not show any significant number of counts in the acquired spectra even at an applied bias of $-1000 \mathrm{~V}$ (electron sensitive transport) and $+550 \mathrm{~V}$ (hole sensitive transport). Higher positive voltages could not be used due to increased noise and instability of the detector baseline even without irradiation. Fig. 4 (a) shows the spectra originating from the white rectangle marked in area B, which can be compared directly to Fig. 3 (a). PHS from all the white marked areas indicated in Fig. 3 (d) have been analysed and the resulting average CCE of the registered pulses as a function of the applied bias voltage is displayed in Fig. 4 (b). A small decrease in signal amplitude during the acquisition of each dataset has been noticeable during the analysis of the data taken from area A, indicating polarisation phenomena. The effect is negligible compared to the overall changes in detector performance in this discussion. In the investigated bias range, the CCE only saturates in area A (received dose of $10^{12} \mathrm{~cm}^{-2}$ ) with $97 \%$ CCE for hole and $93 \%$ CCE for electron transport. 
(a) Area B: after irradiation

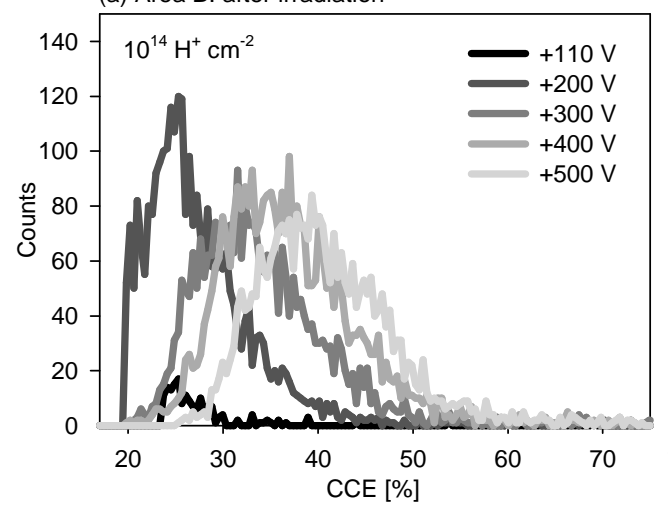

(b) after irradiation

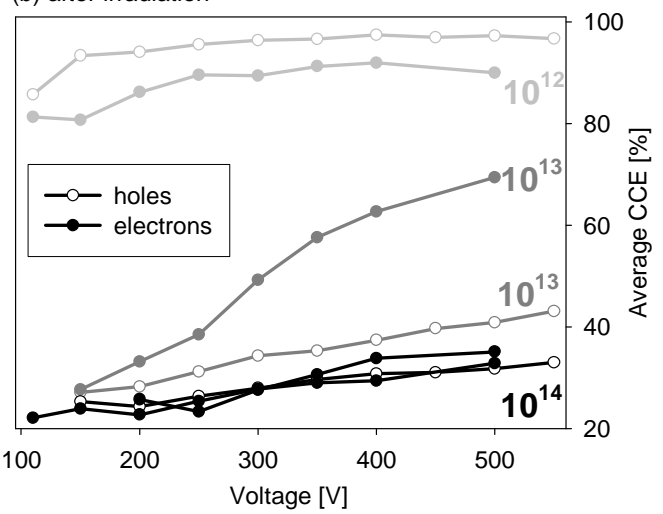

Fig. 4: (a) PHS acquired from the white rectangular area within area B (indicated in Fig. 3 (d)), after irradiation damage was introduced. (b) Average CCE vs Voltage extracted from the white areas A, B, C representing a received dose of $10^{12}, 10^{13}$ and $10^{14} 2.6 \mathrm{MeV}$ protons $\mathrm{cm}^{-2}$ respectively; open symbols represent hole transport, solid circles electrons.

\section{Discussion}

The clear outlines of the irradiated areas observed in the IBIC images, show that the non-irradiated parts of the detector are hardly affected by the introduced damage, which means that diffusion/migration effects of defects are not modifying the electronic properties of the sample over length scales significantly larger than the damaging beam size, i.e. maximum $\sim 100 \mu \mathrm{m}$. This seems small compared to the observed changes in luminescence spectra of alpha particle irradiated natural IIa diamond, which extended as far as $1.5 \mathrm{~mm}$ away from the irradiated area [19].

The contacts were irradiated with the sample and had not been renewed between the IBIC measurements. Thus, it cannot be excluded that some of the observed degradation of detector performance with proton dose is caused by a degradation of contact performance rather then bulk material damage. However, assuming that diffusion effects are negligible, the largest amount of structural damage caused by the proton irradiation in our experiment is concentrated in the so called Bragg peak at the maximum penetration depth of $\sim 40 \mu \mathrm{m}$. The charge carriers, whose movement gives rise to the IBIC signal, are all created in the same volume of material that has been damaged and we assume that the degradation of the electronic transport properties of the device is predominantly limited to the initially damaged volume. At larger depths, the material quality is assumed to be unaltered, providing 'complete' charge collection at sufficient field strength. Charge trapping in the deteriorated material can lead to polarisation and thereby reducing the effective field strength in the undamaged material. Thus, the observed CCE reflects the proportion of charge carriers who are able to escape from the damaged region and are exposed to sufficient electric field strengths to travel through the remaining thickness. This is almost all of them for the lowest proton dose of $10^{12} \mathrm{~cm}^{-2}$. Our values appear significantly lower then the doses published for the onset of strong degradation of charge transport by Pomorski, de Boer [8, 9] and their respective coworkers, using $26 \mathrm{MeV}$ protons. We have calculated the concentrations of vacancies produced by 2.6 $\mathrm{MeV}$ and $26 \mathrm{MeV}$ protons to obtain a rough estimate of the relative amounts of structural damage introduced into the crystal lattice using SRIM (http://www.srim.org) [11]. The vacancy concentration is about 250 times higher at its maximum in the Bragg peak in the $2.6 \mathrm{MeV}$ case than the approximately homogeneous concentration throughout the whole device volume calculated for the $26 \mathrm{MeV}$ irradiations. This illustrates that in our case, the nuclear energy loss per particle, which causes most of the structural damage inside the device volume is much larger and thus primarily responsible for the difference, rather then a difference in radiation hardness of the material. 


\section{Conclusions}

We have used IBIC imaging to investigate the spatial CCE distribution in a high quality synthetic CVD single crystal diamond with $2.6 \mathrm{MeV}$ protons. Initially, a very uniform response of the whole active detector area with $100 \%$ CCE can be achieved at electric field strength $>0.4 \mathrm{~V} . \mu \mathrm{m}^{-1}$, where all small polarisation phenomena are overcome. Subsequently, the device has been damaged with $2.6 \mathrm{MeV}$ protons at doses between $10^{12}$ and $10^{16}$ protons $\mathrm{cm}^{-2}$. The $100 \% \mathrm{CCE}$ was not fully recovered in any of the damaged areas, however for the dose of of $10^{12} \mathrm{~cm}^{-2}$ the signal amplitude saturated at values of $92 \%$ and $97 \%$ for electrons and holes. In contrast, at absorbed doses of more than $5 \times 10^{14}$ protons $\mathrm{cm}^{-2}$, the electronic properties of the damaged volume of the material have been damaged sufficiently to either trap all created charge carriers within it and/or cause sufficient polarisation to reduce the signal amplitude below the detection limit.

Acknowledgements We are thankful for the collaboration with Element Six Ltd, the support of the staff at the Surrey ion beam centre and the funding of the PPARC grant PP/D507607/1.

\section{References}

[1] M. Pomorski, E. Berdermann, A. Caragheorgheopol, M. Ciobanu, M. Kiš, A. Martemiyanov, C. Nebel, and P. Moritz, phys. stat. sol. (a) 203, 3152 (2006).

[2] J. Isberg, J. Hammersberg, E. Johansson, T. Wikström, D. J. Twitchen, A. J. Whitehead, S. E. Coe, and G. A. Scarsbrook, Science 297, 1670 (2002).

[3] P. Bergonzo, D. Tromson, and C. Mer, Semicond. Sci. Technol. 18, S105 (2003).

[4] A. Mainwood, Diamond Relat. Mater. 7, 504 (1998).

[5] A. A. Gippius, R. A. Khmelnitsky, V. A. Dravin, and A. V. Khomich, Diamond Relat. Mater. 12, 538 (2003).

[6] S. Prawer, Diamond Relat. Mater. 4, 862 (1995).

[7] D. Meier, W. Adam, C. Bauer, E. Berdermann, P. Bergonzo, F. Bogani, E. Borchi, M. Bruzzi, C. Colledani, J. Conway, W. Dabrowski, P. Delpierre, A. Deneuville, W. Dulinski, B. Van Eijk, A. Fallou, F. Foulon, M. Friedl, C. Jany, K. K. Gan, E. Gheeraert, E. Grigoriev, G. Hallewell, R. Hall-Wilton, S. Han, F. Hartjes, J. Hrubec, D. Husson, H. Kagan, D. Kania, J. Kaplon, R. Kass, K. T. Knöpfle, M. Krammer, P. F. Manfredi, R. D. Marshall, M. Mishina, F. Le Normand, L. S. Pan, V. G. Palmieri, H. Pernegger, M. Pernicka, A. Peitz, S. Pirillo, K. Pretzl, V. Re, J. L. Riester, S. Roe, D. Roff, A. Rudge, S. Schnetzer, S. Sciortino, V. Speziali, H. Stelzer, R. Stone, R. J. Tapper, R. Tesarek, G. B. Thomson, M. Trawick, W. Trischuk, R. Turchetta, A. M. Walsh, R. Wedening, P. Weilhammer, H. Ziock, and M. Zoeller, Nucl. Instrum. Methods A 426, 173 (1999).

[8] W. de Boer, J. Bol, A. Furgeri, S. Müller, C. Sander, E. Berdermann, M. Pomorski, and M. Huhtinen, phys. stat. sol. (a) 204, 3004 (2007).

[9] M. Pomorski, E. Berdermann, W. de Boer, A. Furgeri, C. Sander, and J. Morse, Diamond Relat. Mater. 16, 1066 (2007).

[10] A. Simon, C. Jeynes, R. P. Webb, R. Finnis, Z. Tabatabaian, P. J. Sellin, M. B. H. Breese, D. F. Fellows, R. van den Broek, R. M. Gwilliam, Nucl. Instrum. Methods B 219-220, 405 (2004).

[11] J. P. Biersack, and L. Haggmark, Nucl. Instr. and Meth. 174 (1-2), 275 (1980).

[12] C. Canali, E. Gatti, S. F. Kozlov, P. F. Manfredi, C. Manfredotti, F. Nava, A. Quirini, Nucl. Instrum. Methods 160, 73 (1979).

[13] J. Kaneko, M. Katagiri, Nucl. Instrum. Methods. A 383, 547 (1996).

[14] J. Morse, M. Salomé, E. Berdermann, M. Pomorski, J. Grant, V. O'Shea, and P. Ilinski, submitted to the Proceedings of Symposium P, MRS Fall Meeting, Boston (2007).

[15] T. Kashiwagi, K. Hibino, H. Kitamura, K. Mori, S. Okuno, T. Takashima, Y. Uchihori, K. Yajima, M. Yokota, and K. Yoshida, IEEE Trans. on Nucl. Science, 53(2), 630 (2006).

[16] G. F. Knoll, Radiation Detection and Measurement, John Wiley \& Sons, Inc., New York, 1999.

[17] V. Kh. Liechtenstein, N. V. Eremin, R. Golser, W. Kutschera, A. A. Paskhalov, A. Priller, P. Steier, C. Vockenhuber, and S. Winkler, Nucl. Instrum. Methods A 521, 203 (2004).

[18] P. J. Sellin, A. Lohstroh, A. W. Davies, A. Galbiati, J. Parkin, S. G. Wang, and A. Simon, Nucl. Instrum. Methods B 260, 293 (2007).

[19] S. Han, S. G. Prussin, J. W. Ager III, L. S. Pan, D. R. Kania, S. M. Lane, and R. S. Wagner, Nucl. Instrum. Methods B 80/81, 1446 (1993). 